\title{
Rotation-Invariant Nonlinear Filters Design
}

\author{
Saúl Martínez-Díaz ${ }^{1}$ and Saúl Martínez-Chavelas ${ }^{2}$ \\ ${ }^{1}$ Instituto Tecnológico de La Paz, División de Estudios de Posgrado e Investigación, \\ Boulevard Forjadores de Baja California Sur No. 4720, \\ La Paz BCS, México \\ ${ }^{2}$ Universidad Nacional Autónoma de México, \\ Facultad de Estudios Superiores Acatlán, Av. Alcanfores S/N, \\ Naucalpan Edo. De México, México
}

\begin{abstract}
Geometrical distortions are a major problem in image recognition. Composite correlation filters can be used for distortion-invariant image recognition by incorporating rotated versions of the target object. Traditionally composite filters are designed with linear techniques; but, these filters are sensitive to non-Gaussian noise. On the other hand, for the same purpose, composite nonlinear filters have been proposed too. These filters have a good discrimination capability and they are robust to non-Gaussian noise and illumination changes; however, the performance of filter could be reduced when the number of training images incorporated increases. In this paper, we propose a method for designing rotation-invariant composite nonlinear filters. The method tries to maximize the number of objects incorporated into the filter and preserve its performance.
\end{abstract}

Keywords: Nonlinear filters, distortion invariance, morphological correlation, composite filters.

\section{Introduction}

Pattern recognition techniques applied to images have been studied intensively over the last years. The main reason for this is the great amount of applications for recognition systems in areas such as medicine, robotics, military, industry and others. Nevertheless, several reasons make difficult the recognition task. Some of them are noise, geometrical distortions of objects to be recognized and other objects in the same scene. Among others, correlation-based methods have been used extensively for this purpose. Some advantages of correlation are shift-invariance and solid mathematical foundation. Besides, correlation methods exploit all information from images in the recognition process. In this case the basic recognition procedure is: Design a template (filter) with one or several training images, correlate the filter with an input test image and establish a threshold at the correlation output. A correlation value greater than threshold indicates that target is located at coordinates of the correlation peak.

Correlation filters can be designed by optimizing some performance criteria with linear techniques. For instance, the classical matched spatial filter (MSF) [1] is 
optimal if an input image is corrupted by additive Gaussian noise. Besides, Synthetic Discriminant Function (SDF) [2], and Minimum Average of Correlation Energy (MACE) [3] filters can be used for distortion-invariant multiclass pattern recognition. In addition, an adaptive approach for the filter design has been proposed [4], in order to reject other objects from scenes. These properties make composite filters suitable for applications such as face recognition $[5,6]$. However, the above mentioned filters use a linear correlation operation. A drawback of linear filters is their sensitive to most kind of real noise.

On the other hand, several approaches of nonlinear filter design have been proposed too $[7,8,9]$. Recently, nonlinear composite filters for distortion-invariant pattern recognition were introduced [10]. The filters are designed as a logical combination of binary objects. Correlation is computed among the filter and a test scene with a nonlinear operation called Morphological Correlation (MC), which is derived from minimization of Mean Absolute Error (MAE) and generates sharper peaks than linear correlation on the output plane [11]. These kinds of filters have demonstrated a good discrimination capability and noise tolerance. Moreover, with the help of threshold decomposition [12], this technique can be applied to grayscale images as well. A drawback of nonlinear correlation is its high computational cost for large images; for this reason, parallel implementations were proposed [13]. However, by incorporating many reference images to the filter could reduce its discrimination capability. Unfortunately, traditional optimization techniques cannot be applied on the composite filter design; therefore a trial and error procedure is needed to incorporate reference images into the template, and check its performance under certain criterion. With the purpose of prevent a low performance when a large number of reference images is available, it might be necessary to design the filters with a subset of the entire set of images. In these cases, if the number of training images is high, the problem of designing the filter by testing all possible combinations of images is computationally untreatable.

In this paper, we propose a method to design composite nonlinear filters for rotation invariant pattern recognition. The mean procedure incorporates reference images with an incremental method that avoids an exhaustive search in the filter design process. The paper is organized as follows: In section 2 we explain nonlinear composite filters. In section 3 we introduce the proposed design method. In section 4 computer simulations are provided and discussed. Section 5 summarizes our conclusions.

\section{$2 \quad$ Nonlinear Filtering}

The nonlinear filtering method is a locally adaptive processing of the signal in a moving window. The moving window is a spatial neighborhood containing pixels surrounding the central window pixel geometrically. The neighborhood is referred to as the W-neighborhood. The shape of the W-neighborhood is similar to the region of support of the target. The size of the neighborhood is referred to as $|W|$, and it is approximately taken as the size of the target. In the case of non-stationary noise or cluttered background (space-varying data), it is assumed that the W-neighborhood is sufficiently small and the signal and noise can be considered stationary over the window area. 


\subsection{Nonlinear Composite Filters}

According to the threshold decomposition concept [11], a gray-scale image $X(k, l)$ can be represented as a sum of binary slices:

$$
X(k, l)=\sum_{q=1}^{Q-1} X^{q}(k, l)
$$

where $\left\{X^{q}(k, l), q=1, \ldots Q-1\right\}$ are binary slices obtained by decomposition of the image with a threshold $q$ as follows

$$
X^{q}(k, l)= \begin{cases}1, \text { if } & X(k, l) \geq q \\ 0, & \text { otherwise }\end{cases}
$$

Now, assume that there are $N$ training objects to be recognized from the true class $\left\{T_{i}(k, l), i=1 \ldots N\right\}$. First, binary images are obtained by threshold decomposition of the training set. Next we construct the non-linear filter $\left(\mathrm{H}_{\mathrm{NSDF}}\right)$ as logical combinations of the binary images as:

$$
H_{N S D F}(k, l)=\sum_{q=1}^{Q-1}\left[\bigcap_{i=1}^{N} T_{i}^{q}(k, l)\right]
$$

where $\left\{T_{i}^{q}(k, l), q=1, \ldots Q-1, i=1, \ldots N\right\}$ are binary slices obtained by threshold decomposition from corresponding training images. $\bigcap$ represents the logical intersection of two planes: the result at coordinates $(k, l)$ is 1 if the corresponding pixels of both planes are equal to 1 ; otherwise, the result is 0 . The neighborhood $W$ is taken as the region of support of the composite filter.

\subsection{Morphological Correlation}

Let $\{T(m, n)\}$ and $\{S(k, l)\}$ be a template and a test scene respectively, both with $Q$ levels of quantization. Here $(m, n)$ and $(k, l)$ are the pixel coordinates. The local nonlinear correlation derived from the MAE criterion between a normalized input scene and a shifted version of the target at coordinates $(k, l)$ can be defined as

$$
C(k, l)=\sum_{m, n \in W} \operatorname{MIN}[a(k, l) S(m+k, n+l)+b(k, l), T(m, n)]
$$

where the sum is taken over the $W$-neighborhood. $a(k, l)$ and $b(k, l)$ are local normalizing coefficients, which take into account unknown illumination and bias of the target, respectively. The optimal coefficients with respect to the MAE can be estimated by minimizing the Mean Squarer Error (MSR) between the window signal and the target. Their explicit estimates are given by: 


$$
\begin{gathered}
a(k, l)=\frac{\sum_{m, n \in W} T(m, n) \cdot S(m+k, n+l)-|W| \cdot \bar{T} \cdot \bar{S}(k, l)}{\sum_{m, n \in W}(S(m+k, n+l))^{2}-|W| \cdot(\bar{S}(k, l))^{2}} \\
b(k, l)=\bar{T}-a(k, l) \cdot \bar{S}(k, l)
\end{gathered}
$$

here $\bar{T}$ and $\bar{S}(k, l)$ are the average of the target and local window signal over the $W$-neighborhood at the $(k, l)$ 'th window position, respectively. Finally, the nonlinear correlation in equation (4) is computed among the test image and the composite filter. The result is normalized by $u / s$. Here $u$ is the desired value at the correlation output, and $s=\sum_{k, l \in W} H_{N S D F}(k, l)$.

\section{$3 \quad$ Filter Design Method}

We wish to design a rotation-invariant nonlinear composite filter with a tradeoff between the number of reference images incorporated and the performance of the filter. The filter must incorporate representative images of the expected distortions; therefore, a bank of images containing rotated versions of target is needed. We are interested in constructing a filter with good performance characteristics for a giving observed scene, i. e. with a fixed set of patterns or a fixed background to be rejected, rather than in constructing a filter with average performance parameters over an ensemble of images; for this reason we optimize with respect to an image, which is representative of the expected background.

We use as a measure of performance the discrimination capability, which is defined as the ability of a filter to distinguish a target from other objects [14]. If a target is embedded into a background that contains both true and false class objects, then the DC can be expressed as follows:

$$
D C=1-\frac{\left|C^{B}(0,0)\right|^{2}}{\left|C^{O}(0,0)\right|^{2}}
$$

where $C^{B}(0,0)$ is the maximum in the correlation plane over the background area to be rejected and $C^{O}(0,0)$ is the maximum in the correlation plane over the area of the object to be recognized. The area of the object to be recognized is determined by the region of support of the target. The background area is complementary to the area of the object to be recognized.

The composite filter design can initiates with a single image as template and incorporating other images one by one. Note that performance can be reduced if two noncompatible images are incorporated in the same filter. Then, if the incorporation of any image reduces the performance of the filter, it is not necessary to test any other combination containing such image in the same filter; in that case the image can be 
discarded. Nonetheless, the discarded image may perhaps be combined with other images and maintain a good performance; therefore all images must be tested as the initial template. For these reasons we propose the following design algorithm:

1. Select the first reference image

2. If some images remain untested, incorporate one of them into the filter

3. Compute the DC

4. If DC is less than a constant factor $\alpha>0$, remove the last incorporated image

5. Go to step 2

In order to allow the combination of non consecutive images, in step 2 the image is selected randomly. Since the reduction of performance may occur because two images are incompatible, the above procedure is repeated initiating with the second image, third image, and so on, until all images are tested as first reference image. Finally, the filter with the maximum of images incorporated is selected. Note that, although the procedure is suboptimal, the number of correlations needed in the design process is reduced from $\mathrm{O}\left(2^{\mathrm{n}}\right)$ to $\mathrm{O}\left(\mathrm{n}^{2}\right)$, for $\mathrm{n}$ reference images.

\section{Computer Simulations}

In this section, by means of computer simulations we illustrate the proposed method. Performance of proposed filters is compared with that of MACE filters, in terms of discrimination capability and robustness to noise. The frequency response of the MACE filter can be expressed as [3]

$$
\mathbf{h}_{M A C E}=\mathbf{D}^{-1} \mathbf{X}\left(\mathbf{X}^{+} \mathbf{D}^{-1} \mathbf{X}\right)^{-1} \mathbf{u}
$$

Where $\mathbf{X}$ is a matrix containing the Fourier transform of training images converted to column vectors in lexicographic order, $\mathbf{D}$ is a diagonal matrix that contains the average power spectrum of the training images and $\mathbf{u}$ is a column vector containing desired output values of correlation, corresponding to each training image. Superscripts + and -1 mean conjugate transpose and inverse matrix, respectively.

Now, 72 training images are generated by rotating the reference image (figure 1) in steps of five degrees. For this experiments we set $\alpha=0.1$. Discrimination capability is computed respect to the background image shown in figure 2. Next the proposed design algorithm is applied. Since a filter is generated initiating with each object, we obtain 72 filters. The best filter incorporates 9 objects with a performance greater than the desired value. In contrast, the worst filter incorporates only 5 objects. With the selected images composite filters are designed and tested. Then, rotated target is embedded into cluttered background. Next, salt and pepper noise is added to scene and discrimination capability is computed. The probability of impulsive noise is incremented from $5 \%$ to $15 \%$. In order to guarantee statistically correct results, 30 realizations of noise were generated and tested in each case, and the results were averaged. Figure 3 shows an example of the test scene corrupted by impulsive noise with 0.15 of noise probability. 


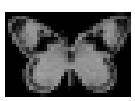

Fig. 1. Reference object used in experiments

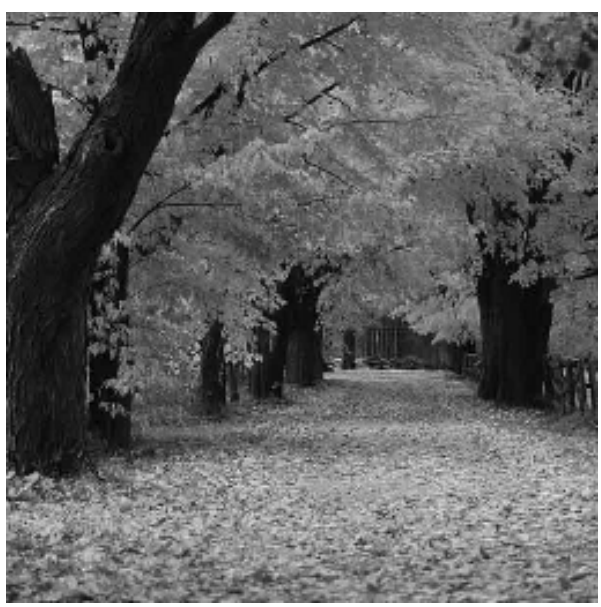

Fig. 2. Representative background used in experiments

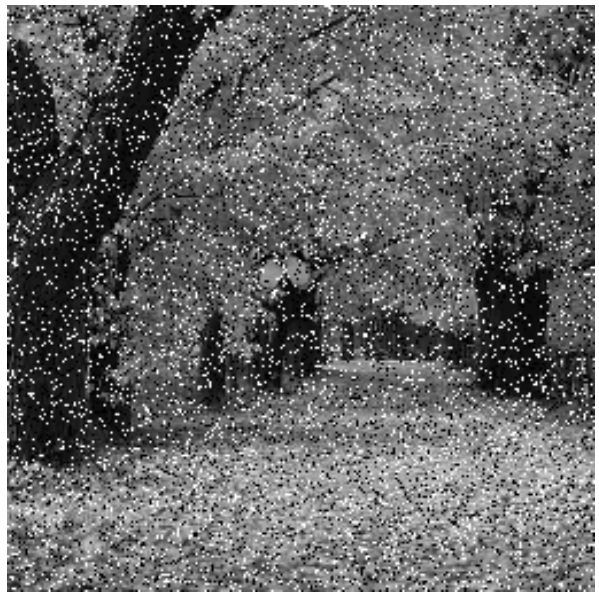

Fig. 3. Test scene corrupted by impulsive noise

Table 1. Results of performance test with impulsive noise

\begin{tabular}{|c|c|c|}
\hline Probability of noise & MACE filter & NSDF filter \\
\hline $5 \%$ & 1 & 1 \\
\hline $7 \%$ & 1 & 1 \\
\hline $9 \%$ & 1 & 1 \\
\hline $11 \%$ & 0 & 1 \\
\hline $13 \%$ & 0 & 1 \\
\hline $15 \%$ & 0 & 1 \\
\hline
\end{tabular}


Table 1 shows the obtained results. A value of one indicates success and a value of zero indicates that filter was unable to identify the target. Note that NSDF filter is able to identify the target in all cases but MACE filter fails when scenes are corrupted by a probability of noise superior to $10 \%$. Finally, salt and pepper noise is fixed at $5 \%$ of probability and zero-mean Gaussian noise is added. Standard deviation of Gaussian noise is varied from 5 to 20 . Figure 4 shows an example of the test scene corrupted by impulsive noise with 0.5 of probability and standard deviation of 20. Table 2 shows results. As can be seen nonlinear filter identify the target in all cases but linear filter fails with high noise.

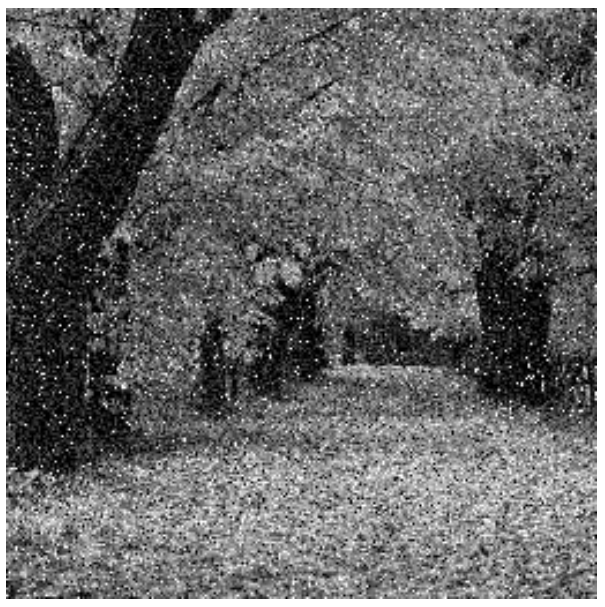

Fig. 4. Test scene corrupted with mixed noise

Table 2. Results of performance test with mixed impulsive and Gaussian noise

\begin{tabular}{|c|c|c|}
\hline Standard deviation of Gaussian noise & MACE filter & NSDF filter \\
\hline 5 & 1 & 1 \\
\hline 10 & 0 & 1 \\
\hline 15 & 0 & 1 \\
\hline 20 & 0 & 1 \\
\hline
\end{tabular}

\section{Conclusions}

In this paper, a rotation-invariant nonlinear composite filters design method was proposed. The filters are designed as a logical combination of a given set of training images. This kind of filters are robust to non-Gaussian noise and illumination changes. The proposed algorithm reduces the number of correlations needed in the design process from $\mathrm{O}\left(2^{\mathrm{n}}\right)$ to $\mathrm{O}\left(\mathrm{n}^{2}\right)$. Computer simulations show robustness to noise of the designed filters even with highly corrupted scenes. 


\section{References}

1. VanderLugt, A.B.: Signal Detection by Complex Filtering. IEEE Trans. Inf. Theory 10, $135-139$ (1964)

2. Hester, C.F., Casasent, D.: Multivariant Technique for Multiclass Pattern Recognition. Appl. Opt. 19, 1758-1761 (1980)

3. Mahalanobis, A., Vijaya-Kumar, B.V.K., Casasent, D.: Minimum Average Correlation Energy Filters. Appl. Opt. 31, 1823-1833 (1987)

4. González-Fraga, J.A., Kober, V., Álvarez-Borrego, J.: Adaptive Synthetic Discriminant Function Filters for Pattern Recognition. Opt. Eng. 45, 057005 (2006)

5. Banerjee, P.K., Datta, A.K.: Generalized Regression Neural Network Trained Preprocessing of Frequency Domain Correlation Filter for Improved Face Recognition and its Optical Implementation. Optics \& Laser Technology 45, 217-227 (2013)

6. Banerjee, P.K., Datta, A.K.: Class Specific Subspace Dependent Nonlinear Correlation Filtering for Illumination Tolerant Face Recognition. Pattern Recognition Letters 36, 177185 (2014)

7. Doh, Y.H., Kim, J.C., Kim, J.W., Choi, K.H., Kim, S.J., Alam, M.S.: Distortion-invariant Pattern Recognition based on a Synthetic Hit-Miss Transform. Opt. Eng. 43, 1798-1803 (2004)

8. Wang, Q., Deng, Y., Liu, S.: Morphological Fringe-Adjusted Joint Transform Correlation. Opt. Eng. 45, 087002 (2006)

9. García-Martínez, P., Tejera, M., Ferreira, C., Lefebvre, D., Arsenault, H.H.: Optical Implementation of the Weighted Sliced Orthogonal Nonlinear Generalized Correlation for Non uniform Illumination Conditions. Appl. Opt. 41, 6867-6874 (2002)

10. Martínez-Díaz, S., Kober, V.: Nonlinear Synthetic Discriminant Function Filters for Illumination-Invariant Pattern Recognition. Opt. Eng. 47, 067201 (2008)

11. Maragos, P.: Morphological Correlation and Mean Absolute Error Criteria. In: Proc. Conf. IEEE Trans. Acoust. Speech Signal Process., pp. 1568-1571 (1989)

12. Fitch, J.P., Coyle, E.J., Gallagher Jr., N.C.: Median Filtering by Threshold Decomposition. IEEE Trans. Acoust. Speech Signal Process., 1183-1188 (1984)

13. Martínez-Díaz, S.: Parallel Architecture for Binary Image Recognition. In: Proceedings of the World Congress on Engineering, London (2012)

14. Yaroslavsky, L.P.: The Theory of Optimal Methods for Localization of Objects in Pictures. In: Progress in Optics XXXII, pp. 145-201. Elsevier (1993) 